\title{
Estudo da Recuperação das Propriedades de Poliestireno de Alto Impacto (HIPS) através da Incorporação de Borracha Termoplástica Tipo Estireno-Butadieno-Estireno (SBS)
}

\author{
Vanda F. Ribeiro \\ Softer Brasil Compostos Termoplásticos Ltda. \\ Nei S. Domingues Jr., Izabel C. Riegel \\ Instituto de Ciências Exatas e Tecnológicas, Feevale
}

\begin{abstract}
Resumo: A tenacificação de termoplásticos vítreos através da adição de partículas de borracha na matriz polimérica é um processo amplamente estudado. Neste trabalho foi avaliada a recuperação das propriedades de poliestireno de alto impacto (HIPS) reciclado através da incorporação de copolímeros estireno-butadieno-estireno (SBS) de estrutura linear e radial. As blendas foram processadas em uma extrusora dupla rosca. Os copolímeros foram adicionados em teores de 5, 10 e $15 \%$ em massa. As propriedades avaliadas foram o índice de fluidez, a morfologia e o comportamento frente a ensaios de tração, flexão e resistência ao impacto das blendas formuladas. Em todos os casos, o SBS radial foi mais eficiente na recuperação das propriedades do poliestireno de alto impacto que o SBS linear quando comparadas às propriedades do HIPS reciclado.
\end{abstract}

Palavras-chave: HIPS, reciclagem, tenacificação, copolímero SBS.

\section{Recovering Properties of Recycled HIPS Through Incorporation of SBS Triblock Copolymer}

\begin{abstract}
Toughening glassy thermoplastics by blending a rubbery material to the polymeric matrix has been extensively studied. In this work, we evaluated the resulting properties of recycled high impact polystyrene (HIPS), after the incorporation of either radial or linear SBS triblock copolymers. The blends were melt mixed using a twin-screw extruder. The copolymers were added at 5,10 and $15 \%$ weight percent. The investigated properties included the determination of the melt flow index, the morphology and the mechanical behavior according to standard tension, flexion and impact methods of all formulated blends. In all cases, the radial SBS was more efficient in recovering the HIPS properties than the linear SBS, when comparing the properties of the blends with the properties of recycled HIPS.
\end{abstract}

Keywords: HIPS, recycling, toughening, SBS copolymer.

\section{Introdução}

O HIPS (poliestireno de alto impacto - do inglês high impact polystyrene) é uma blenda imiscível de poliestireno (PS), polibutadieno (PB) e uma determinada concentração por enxertia dos dois componentes ${ }^{[1]}$. O HIPS é obtido pela polimerização in situ do estireno na presença de polibutadieno formando domínios discretos da fase elastomérica dispersos em uma matriz contínua de $\mathrm{PS}^{[2]}$. A polimerização elastômero-estireno gera um produto com maior resistência ao impacto que o poliestireno homopolímero $^{[3,4]}$ e blendas pós-reator de PS e elastômero ${ }^{[3]}$. O HIPS é também mais resistente a líquidos orgânicos, óleos e graxas que o PS sem adição de $\mathrm{PB}^{[4]}$. Por sua maior tenacidade, o HIPS é amplamente utilizado em aplicações que necessitem maior resistência que o PS não-tenacificado, tais como gabinetes de refrigeradores, eletroeletrônicos, peças de máquinas e veículos, grades de ar-condicionado, saltos para calçados, brinquedos, acessórios para móveis, embalagens de proteção contra choques, utilidades domésticas, entre outros ${ }^{[2,3]}$.

A reciclagem de plásticos é uma prática consolidada e constitui-se uma alternativa real para a redução da demanda por matérias-primas petroquímicas, contribuindo para a preservação dos recursos naturais não renováveis e para o desenvolvimento sustentável. Segundo a ABIPLAST, Associação Brasileira da Indústria do Plástico, em 2009, foram consumidos no Brasil 5,5 milhões de toneladas de plásticos virgem, ou seja, não reprocessados, sendo que $7 \%$ deste valor corresponderam a resinas de poliestireno ${ }^{[5]}$, entre elas o poliestireno de alto impacto (HIPS). Os processos de reciclagem são genericamente classificados em quatro categorias: primária, secundária, terciária e quaternária ${ }^{[6,7]}$. Na reciclagem primária, a matéria-prima provém de fonte confiável e limpa, como no caso de resíduos da indústria de plásticos e o produto final tem características equivalentes àquelas dos produtos originais produzidos com material virgem. $\mathrm{Na}$ reciclagem secundária, a matéria-prima provém de resíduos sólidos urbanos e produto o final geralmente tem propriedades inferiores aos produtos produzidos com material virgem. A reciclagem terciária tem como base a despolimerização, gerando oligômeros, monômeros e substâncias de baixa massa molecular, que poderão passar por nova polimerização, processamento, industrialização e uso. A reciclagem quaternária consiste na incineração controlada de resíduos poliméricos, visando o aproveitamento de seu conteúdo energético ${ }^{[6,7]}$. No Brasil, a reciclagem primária é a mais desenvolvida, absorvendo $5 \%$ do plástico consumido no país ${ }^{[8]}$. Processos mecânicos de reciclagem tem sido identificados como preferíveis para a gestão de resíduos plásticos, pois implica na minimização do consumo de energia e emissão dos gases que contribuem para o aquecimento global ${ }^{[9,10]}$.

Materiais poliméricos tendem a sofrer alterações químicas durante o seu processamento, vida útil e reciclagem, ou pela

Autor para correspondência: Izabel Cristina Riegel, Departamento de Química, Universidade Federal do Paraná - UFPR,

Rua Francisco H. dos Santos, s/n, Centro Politécnico, Jardim das Américas, CP 19081, CEP 81531-980, Curitiba, PR, Brasil, e-mail: izabel.riegel@ufpr.br 
presença de contaminantes que podem alterar a composição, os mecanismos de estabilização e as propriedades mecânicas dos polímeros reciclados. O entendimento dos efeitos dos processos de degradação na vida útil e na subsequente reciclagem é necessário para predizer a durabilidade de novos produtos fabricados com o material reciclado. Pesquisas recentes identificam três propriedades chave como necessárias à avaliação da propriedade de plásticos reciclados e a garantia da sua aplicabilidade na fabricação de novos produtos: o grau de mistura (composição), o grau de degradação, e a presença de compostos de baixo peso molecular ${ }^{[10]}$. O grau de mistura (composição) está relacionado com a presença de impurezas poliméricas no polímero reciclado, como resultado de resíduos de baixa qualidade ou separação deficiente na planta de reciclagem ${ }^{[10]}$. A mistura de diferentes tipos de polímeros frequentemente envolve a perda de propriedades mecânicas do material, principalmente pela incompatibilidade dos polímeros da molecular (contaminantes, aditivos e produtos de degradação) presentes nos polímeros reciclados é essencial para verificar se os produtos reciclados atendem à legislação referente à presença de substâncias perigosas ou restritas. O grau de degradação considera os processos degradativos aos quais cada polímero foi submetido no processamento, uso e posterior recuperação ${ }^{[10]}$, podendo ser oriundo de um processo de despolimerização, oxidação, reticulação ou cisão de ligações químicas ${ }^{[1]}$. A história térmica do material polimérico influencia de forma acentuada a sua estabilidade e degradação.

$\mathrm{O}$ poliestireno de alto impacto é um material adequado a processos de reciclagem mecânica, uma vez que suas propriedades não são significativamente afetadas após múltiplos ciclos de reprocessamento ${ }^{[9,12]}$, entretanto, suas propriedades mecânicas são afetadas por processos termo-oxidativos, sendo a suscetibilidade do poliestireno a foto-degradação ${ }^{[1,13]}$, com resultante queda da tenacidade, um limitante ao uso do HIPS reciclado em aplicações que exijam maior resistência mecânica.

Os elastômeros termoplásticos são compostos que fluem como termoplásticos nas temperaturas de transformação e tem comportamento elástico nas condições de aplicação. Os copolímeros triblocos de estireno-butadieno-estireno (SBS), são exemplos de elastômeros termoplásticos, onde $\mathrm{B}$ representa o polibutadieno e $\mathrm{S}$ representa o poliestireno. Com $\mathrm{Tg}$ em torno $100{ }^{\circ} \mathrm{C}$, os blocos de poliestireno são responsáveis pelo comportamento termoplástico do copolímero, e os blocos de polibutadieno, que possuem $\mathrm{Tg}$ em torno de $-90{ }^{\circ} \mathrm{C}$, são responsáveis pelo comportamento elastomérico ${ }^{[14]}$. Os copolímeros em bloco de estireno-butadieno, especialmente os triblocos, por apresentarem propriedades similares a borracha vulcanizada, ou seja, alta resistência a tração, alta elongação na ruptura e rápida recuperação, tem alto potencial como modificadores de impacto do poliestireno ${ }^{[15,16]}$.

A incorporação de elastômeros como modificadores de impacto é um dos métodos mais utilizados para a melhoria de tenacidade de polímeros frágeis e pode dar-se por mistura mecânica ou por polimerização do polímero vítreo na presença do componente elastomérico, obtendo-se um copolímero do tipo enxertado. São condições essenciais para a obtenção de misturas com alta resistência ao impacto: (a) a temperatura de transição vítrea do componente elastomérico deve ser bem abaixo da temperatura ambiente; (b) a borracha deve formar uma segunda fase dispersa na matriz vítrea; (c) deve haver uma boa adesão entre as fases ${ }^{[17]}$.

Neste trabalho, avaliou-se a influência do teor e do tipo de copolímero estireno-butadieno-estireno (SBS), adicionado como agente modificador de impacto, na recuperação de propriedades mecânicas de um HIPS já submetido à reciclagem primária. Foram preparadas misturas de HIPS reciclado com diferentes teores de copolímeros SBS de estrutura radial e linear e sua caracterização efetuada através da avaliação do índice de fluidez e de propriedades blenda resultante ${ }^{[11]}$. A identificação de compostos de baixo peso

mecânicas. A calorimetria exploratória diferencial (CED) foi utilizada para avaliar a presença de contaminantes poliméricos no material reciclado. A decomposição térmica das composições foi analisada por termogravimetria (TG) e a morfologia das superfícies fraturadas foi caracterizada por microscopia eletrônica de varredura (MEV).

\section{Experimental}

\section{Materiais}

Foram utilizados neste estudo uma resina de poliestireno alto impacto reciclado, proveniente da indústria de eletroeletrônicos, já submetida a processo de reciclagem primária, e dois tipos de copolímero SBS, fabricados pela Kraton Polymers, um com estrutura radial e outro com estrutura linear, com iguais teores de polibutadieno. As principais informações dos materiais utilizados estão listados na Tabela 1.

\section{Preparo das amostras}

As amostras foram preparadas a partir do HIPS reciclado adicionando-se concentrações de 5, 10 ou $15 \%$ de copolímero modificador de impacto, linear ou radial, identificadas como 5, 10 e $15 \mathrm{~L}$ e 5, 10 e 15R respectivamente. Para fins de comparação, foi preparada uma amostra de HIPS sem adição de modificador de impacto (HIPS base). Todas as amostras receberam 0,03\% em massa do antioxidadante 2,6-di-terc-butil-4-metilfenol.

\section{Processamento}

As amostras foram processadas em uma extrusora dupla rosca, corotante, marca Maris, modelo MT $45 \mathrm{~V}(\mathrm{~L} / \mathrm{D}=40$, diâmetro $=45 \mathrm{~mm})$, com nove zonas de temperatura que variaram entre 100 e $220{ }^{\circ} \mathrm{C}$ ao longo do barril. Os parâmetros de processamento foram mantidos constantes, com taxa de alimentação de $45 \mathrm{~kg} / \mathrm{h}$, velocidade de rotação de $250 \mathrm{rpm}$ e $227^{\circ} \mathrm{C}$ de temperatura.

\section{Confecção dos corpos de prova}

Os corpos de prova foram obtidos por moldagem por injeção em uma injetora, da marca Himaco MI 300-100, utilizando os materiais previamente secos em estufa por um período de 6 horas a $70{ }^{\circ} \mathrm{C}$. Os parâmetros de injeção adotados foram 40 bar de pressão de

Tabela 1. Informações do HIPS reciclado e dos copolímeros SBS.

\begin{tabular}{|c|c|c|c|c|}
\hline Propriedade & Norma & $\begin{array}{c}\text { HIPS } \\
\text { reciclado }^{(a)}\end{array}$ & $\begin{array}{c}\text { SBS } \\
\text { linear }^{(a)}\end{array}$ & $\begin{array}{c}\text { SBS } \\
\text { radial }^{(a)}\end{array}$ \\
\hline Estrutura & - & - & Linear & Radial \\
\hline $\begin{array}{l}\text { Teor de polibutadieno } \\
\text { (\% em massa) }\end{array}$ & - & - & 70 & 70 \\
\hline $\begin{array}{l}\text { Teor de estireno } \\
\text { (\% em massa) }\end{array}$ & - & - & 30 & 30 \\
\hline $\begin{array}{l}\text { Resistência ao impacto } \\
\text { IZOD }\left(\mathrm{J}^{\left.-\mathrm{m}^{-1}\right)}\right.\end{array}$ & ASTM D 256 & 24,6 & - & - \\
\hline $\begin{array}{l}\text { Resistência à tração } \\
(\mathrm{MPa})\end{array}$ & ASTM D 638 & 21,9 & - & - \\
\hline $\begin{array}{l}\text { Elongação na ruptura } \\
\qquad(\%)\end{array}$ & ASTM D 638 & 4,5 & - & - \\
\hline $\begin{array}{c}\mathrm{MFI}^{(\mathrm{b})}(\mathrm{g} / 10 \mathrm{~min}) \\
\left(200^{\circ} \mathrm{C} / 5 \mathrm{~kg}\right)\end{array}$ & ASTM D 1238 & 7,6 & 6 & $<1$ \\
\hline $\begin{array}{c}\text { Massa molecular }{ }^{(\mathrm{c})} \bar{M}_{w} \\
\left(\mathrm{~g} \cdot \mathrm{mol}^{-1}\right)\end{array}$ & & - & 124.000 & 320.000 \\
\hline Densidade $\left(\mathrm{g} . \mathrm{cm}^{-3}\right)$ & ASTM D 792 & 1,04 & 0,94 & 0,94 \\
\hline \multicolumn{5}{|c|}{$\begin{array}{l}\text { (a) valores determinados pelos autores; (b) MFI: melt flow index; } \\
\text { and (c) Determinados por Cromatografia de Exclusão por Tamanhos } \\
\text { em equipamento Viscotek, modelo } 270 \text { Triple Detector. Amostras } \\
\text { solubilizadas em THF. }\end{array}$} \\
\hline
\end{tabular}


injeção, 10 bar de contra-pressão, 20 segundos de tempo de injeção, 40 segundos de resfriamento, temperatura do molde em $40{ }^{\circ} \mathrm{C} \mathrm{e}$ perfil de temperatura $180 / 185 / 185^{\circ} \mathrm{C}$.

\section{Caracterização das amostras}

Os ensaios de tração uniaxial e flexão em três apoios foram realizados em uma Máquina Universal de Ensaios EMIC DL 2000. Para tração adotou-se a norma ASTM D 638 com velocidade de afastamento de $10 \mathrm{~mm} / \mathrm{min}$. $\mathrm{O}$ teste de flexão em três apoios foi realizado conforme ASTM D 790, com 64 mm de distância entre os apoios e taxa de deformação de $1,6 \mathrm{~mm} / \mathrm{min}$.

A resistência ao impacto IZOD foi ensaiada conforme ASTM D 256, corpos de prova com entalhe, em um equipamento EMIC, modelo AIC-1. O índice de fluidez foi determinado conforme ASTM D 1238, na condição de $200{ }^{\circ} \mathrm{C} / 5 \mathrm{~kg}$, em um plastômetro marca CEAST, modelo Melt Flow Junior.

A morfologia das blendas foi estudada por microscopia eletrônica de varredura (MEV) através da observação da superfície de fratura de amostras submetidas ao ensaio de impacto, após seu recobrimento com ouro. Foi utilizado um equipamento da marca JEOL, modelo 6060, com tensão de aceleração $20 \mathrm{kV}$, no Centro de Microscopia da UFRGS.

Termogravimetria foi a técnica de análise adotada para avaliar a estabilidade térmica das composições bem como o teor de umidade, voláteis e resíduos não orgânicos. Os ensaios foram conduzidos na Central Analítica da Feevale, em um equipamento Shimadzu TGA-50, seguindo a norma ASTM D6370. A taxa de aquecimento foi de $10{ }^{\circ} \mathrm{C} / \mathrm{min}$, da temperatura ambiente até $800{ }^{\circ} \mathrm{C}$, em atmosfera de nitrogênio. Os ensaios de calorimetria exploratória diferencial (CED) foram realizados em um equipamento Shimadzu DSC-60, seguindo a norma ASTM D3418. A taxa de aquecimento foi de $20{ }^{\circ} \mathrm{C} / \mathrm{min}$, em dois ciclos de aquecimento. O primeiro aquecimento foi efetuado da temperatura ambiente até $200{ }^{\circ} \mathrm{C}$, mantendo-se cada mostra nesta temperatura por 10 minutos. Os eventos térmicos das amostras ensaiadas foram determinados no segundo aquecimento, que foi realizado na faixa de temperatura de -40 a $250{ }^{\circ} \mathrm{C}$.

\section{Resultados e Discussão}

\section{Propriedades do HIPS reciclado sem adição de SBS}

O valor da resistência ao impacto Izod do HIPS reciclado utilizado nesse trabalho, sem a adição de SBS foi de 24,6 J.m ${ }^{-1}$ (Tabela 1) sendo que um valor típico de resistência ao impacto de HIPS para uso em eletro-eletrônicos é de $110 \mathrm{~J} . \mathrm{m}^{-1[18]}$. A baixa resistência ao impacto é um indicativo de acentuada degradação do material e/ou existência de mistura de outros tipos de poliestireno ${ }^{[19]}$.

A presença de frações de outros polímeros como contaminantes em poliestireno de alto impacto reciclado e a presença de produtos de degradação, podem afetar significativamente o desempenho do material ${ }^{[20]}$. Ensaios de calorimetria exploratória diferencial em mostras de HIPS reciclado reportam que, além do sinal característico da transição vítrea do poliestireno, em torno de $90{ }^{\circ} \mathrm{C}$, pequenos picos de fusão em torno de $120{ }^{\circ} \mathrm{C}$ e/ou $160{ }^{\circ} \mathrm{C}$ podem ser encontrados, indicando a presença da contaminação com polietileno e/ou polipropileno, respectivamente ${ }^{[11,19]}$. A Figura 1 mostra a curva de CED para a amostra de HIPS sem adição de copolímero. A detecção de um único evento térmico a $101,1{ }^{\circ} \mathrm{C}$, característico da temperatura de transição vítrea da fase PS, indica a ausência de polímeros contaminantes como PE e PP. Villaplana et al. ${ }^{[9]}$ observaram em amostras de HIPS, submetido a até nove ciclos de reprocessamento, temperaturas de transições vítrea do PS variando entre 100 e $103{ }^{\circ} \mathrm{C}$, igualmente sem a presença de eventos de fusão.

Estudos sobre a degradação termo-oxidativa de poliestireno de alto impacto reportam uma queda de $85 \%$ na elongação na ruptura após quatro dias de envelhecimento acelerado a $90{ }^{\circ} \mathrm{C}$, com efeitos mais severos que os encontrados no mesmo material submetido a repetidos ciclos de reprocessamento por extrusão ${ }^{[12]}$. Avaliando a degradação de PS e HIPS, submetido a envelhecimentos natural e artificial, Borrelly ${ }^{[13]}$ verificou no HIPS uma drástica redução na elongação e redução intensa na resistência ao impacto durante os primeiros 15 dias de envelhecimento devido, principalmente, à degradação do polibutadieno. A fase polibutadieno, que contem ligações duplas $\mathrm{C}=\mathrm{C}$, é reportada como sendo os sítios iniciais de

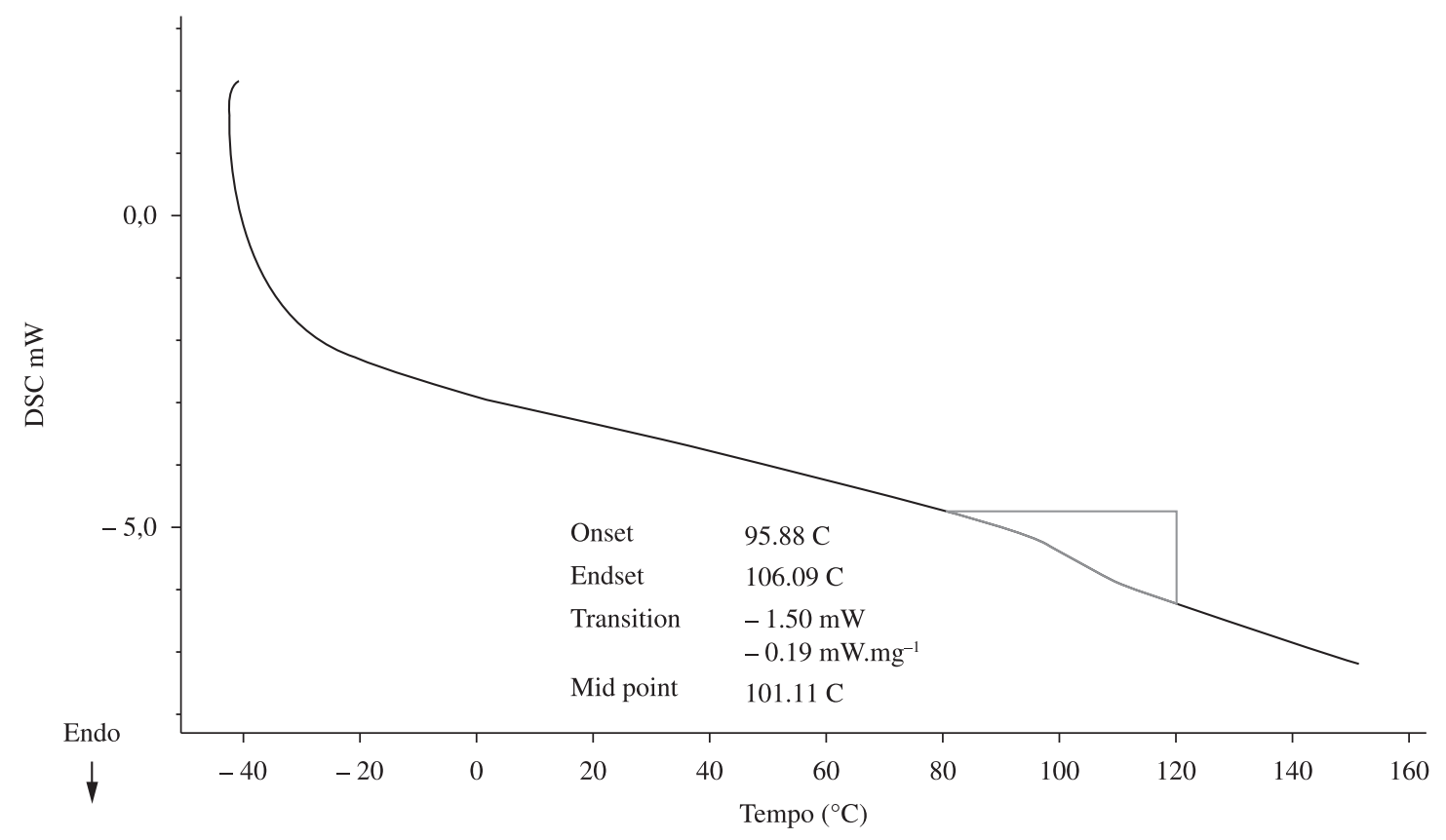

Figura 1. Curva CED do HIPS reciclado, sem adição de SBS. 
processos foto-oxidativos, termo-oxidativos e termo-mecânicos de degradação de copolímeros estirênicos, induzindo alterações na estrutura química ${ }^{[1,9,10]}$. Estes resultados demonstram a importância do conhecimento da procedência e das condições de processamento e estocagem do HIPS reciclado, visto que a mistura com tipos diferentes de poliestireno (não tenacificados), contaminações e condições de armazenamento inadequadas, tais como exposição ao sol e o calor, são fatores determinantes nas propriedades mecânicas e na sua estabilidade à degradação.

Visto que não há presença de outros polímeros no HIPS utilizado e que não se tem conhecimento da história anterior do material, sugere-se que a baixa resistência ao impacto possa ter advindo de degradações termo-oxidativas a que o material tenha sido sujeito anteriormente.

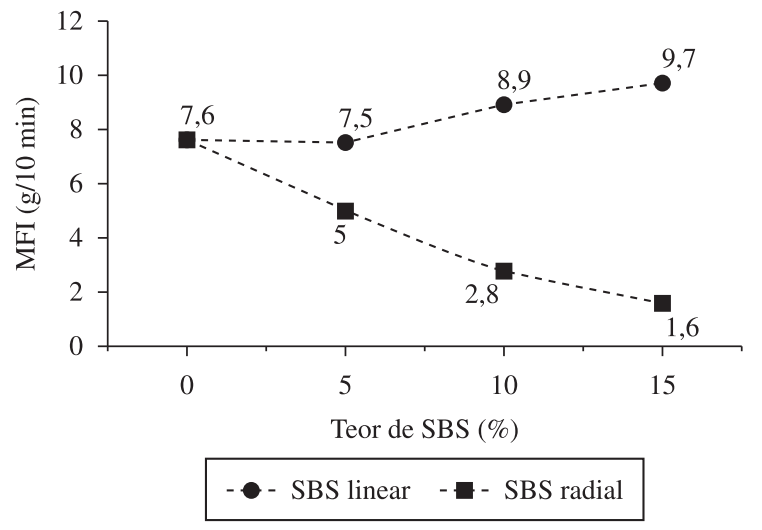

Figura 2. MFI das blendas HIPS reciclado/SBS em função do teor e microstrutura do SBS.

\section{Propriedades das blendas HIPS reciclado/SBS}

\section{Índice de fluidez}

Na Figura 2 encontram-se os resultados de índice de fluidez em função do teor e da microestrura do SBS adicionado ao HIPS reciclado base. O aumento do teor de SBS radial nas composições provocou uma redução do índice de fluidez, devido à maior massa molecular e à sua estrutura radial. Efeito inverso foi observado nas misturas com SBS linear, com exceção da amostra com $5 \% \mathrm{~m} / \mathrm{m}$, que manteve o índice de fluidez do HIPS base. A incorporação de $15 \% \mathrm{~m} / \mathrm{m}$ de SBS linear levou a um incremento de quase $30 \%$ do valor do ínidice de fluidez em relação ao HIPS base. Este aumento do índice de fluidez das composições com 10 e 15\% de SBS linear, estão possivelmente relacionados à cisão das cadeias poliméricas durante o processamento, levando à redução da massa molecular. Comportamento semelhante foi observado com aumento de MFI em HIPS submetidos a ciclos de reprocessamento mecânico ${ }^{[12]}$.

\section{Propriedades mecânicas}

Em todos os teores empregados os valores de resistência ao impacto das misturas HIPS reciclado/SBS radial foram maiores que os encontrados para as misturas com os mesmos teores de SBS linear (Figura 3) e todos superiores ao HIPS reciclado sem adição de SBS. Os resultados superiores nas amostras utilizando SBS radial podem estar relacionados com a maior massa molecular do copolímero e à sua estrutura tipo estrela, que propicia um maior volume livre entre as moléculas e espaço para movimentação intermolecular ${ }^{[15]}$.

Resultados anteriores relatam que o comportamento de polímeros tenacificados com elastômeros está relacionado com a sua morfologia, tamanho médio e distribuição de tamanho das partículas e teor de elastômero ${ }^{[2,15,17]}$. A resistência à tração e o módulo de flexão de um composto depende, principalmente, das propriedades em tensão e flexão dos componentes individuais e da magnitude do trabalho de adesão interfacial.

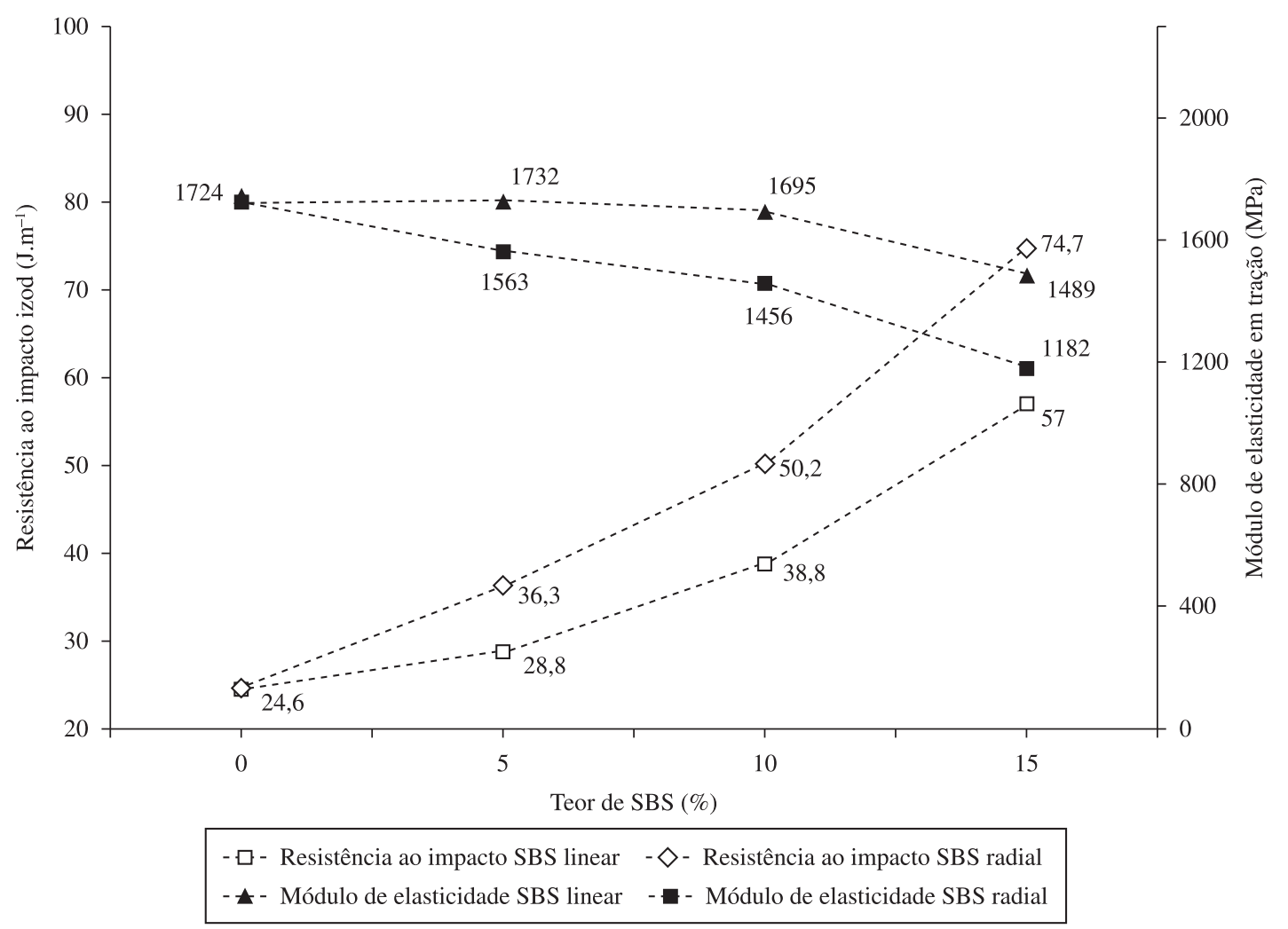

Figura 3. Resistência ao impacto e módulo de elasticidade em tração das blendas HIPS reciclado/SBS. 
Os valores de módulo de elasticidade em tração reduziram com o aumento do componente elastomérico, sendo menores para as composições com SBS radial (Figura 3 e Tabela 2). A mistura $15 \mathrm{R}$ foi a que apresentou maior queda no módulo de elasticidade, indicando menor rigidez de acordo com o aumento da tenacidade observado no ensaio de resistência ao impacto. A Figura 4 mostra os valores de resistência ao impacto das amostras de HIPS reciclado, e das composições com $15 \%$ de SBS, comparadas ao valor típico de resistência ao impacto de referências comerciais de HIPS, destinadas a aplicações de uso geral, tais como brinquedos, utilidades domésticas e acessórios para móveis ${ }^{[21,22]}$.

Os resultados dos ensaios de tração e flexão, para todas as amostras, estão apresentados na Tabela 2, e corroboram os dados obtidos nos ensaios de resistência ao impacto. A adição dos copolímeros SBS provocou o aumento da elongação na ruptura, mas não afetou significativamente a resistência à tração, que em todos os materiais representou uma região de escoamento.

Devido ao baixo módulo da fração elastomérica, a adição de copolímero SBS provocou a diminuição na resistência à flexão e no

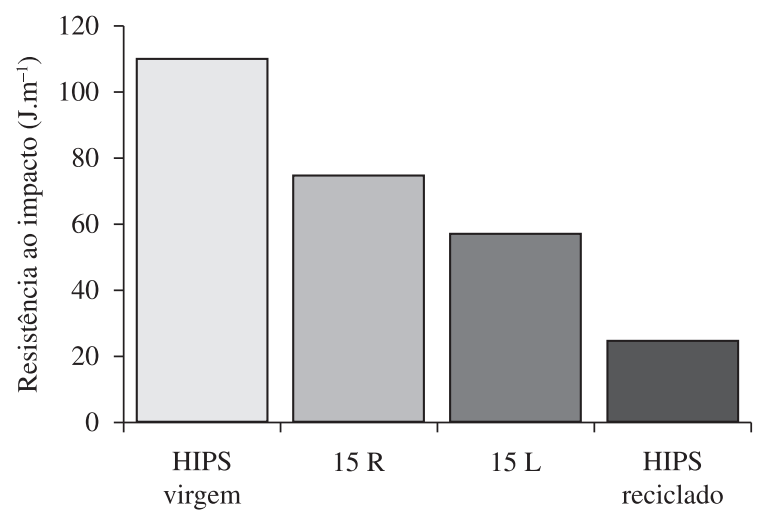

Figura 4. Valores da resistência ao impacto das composições com 15\% de SBS radial (15R) e linear (15L), do HIPS reciclado e do HIPS virgem comercial. módulo de flexão, apontado pela diminuição gradativa da rigidez das blendas com aumento do teor de SBS. As composições preparadas com SBS radial apresentaram valores menores de resistência à flexão e módulo de flexão, quando comparadas às composições com mesmos teores de SBS linear.

\section{Termogravimetria}

Termogravimetria foi utilizada para avaliar a estabilidade térmica, analisar o teor de umidade, voláteis e resíduos não orgânicos nas blendas de HIPS reciclado/SBS. Para todas as composições foram determinadas a $\mathrm{T}_{0,1}$ que é a temperatura em que ocorre $10 \%$ de perda de massa, tida como a temperatura inicial do processo de degradação, e a $\mathrm{T}_{0.5}$ que é a temperatura onde ocorre $50 \%$ da perda de massa (Tabela 3). Conforme esperado, as formulações analisadas apresentaram comportamentos de decomposição térmica intermediários às curvas de decomposição dos componentes individuais. Observa-se que os copolímeros SBS apresentam estabilidade térmica relativa superiores ao HIPS base reciclado. Em todas as amostras testadas não foram verificadas perdas de massa na faixa de temperatura entre 25 e $150{ }^{\circ} \mathrm{C}$, indicando que os materiais não apresentaram quantidades significativas de umidade ou outras substâncias voláteis nesta faixa de temperatura. As blendas com SBS radial apresentaram estabilidade térmica superior em aproximadamente $10^{\circ} \mathrm{C}$ às blendas com igual teor de SBR linear, mais uma vez influenciados pelo maior peso molecular do primeiro.

$\mathrm{O}$ teor de material inorgânico foi determinado através do percentual de resíduo a $600{ }^{\circ} \mathrm{C}$. Os valores encontrados podem estar relacionados com a presença de aditivos, cargas e pigmentos inorgânicos, resíduos de carbono ou negro de fumo, que tipicamente não se decompõem sob atmosfera normal de nitrogênio.

\section{Morfologia}

A Figura 5 mostra fotomicrografias das superfícies fraturadas no ensaio de resistência ao impacto das amostras de HIPS reciclado, das composições com $15 \%$ de SBS linear e radial, e sem a adição de SBS, observadas por MEV. As imagens das composições com

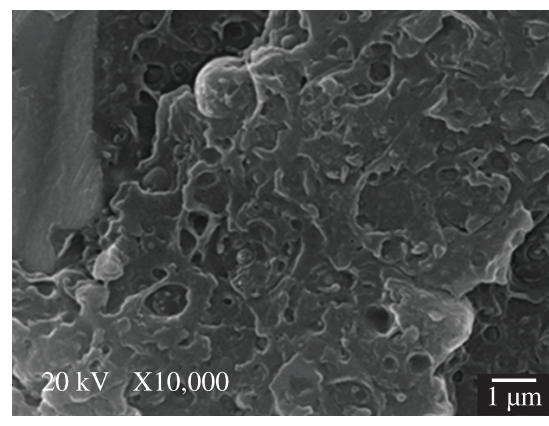

(a)

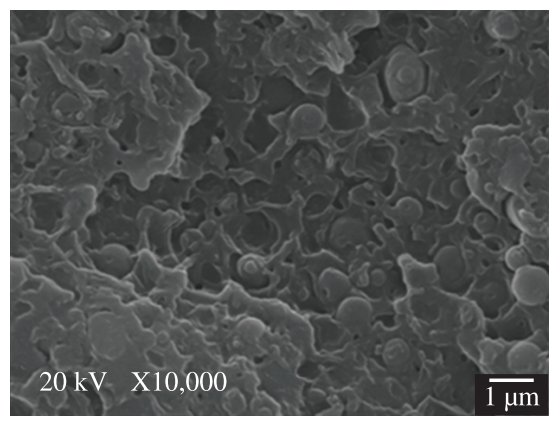

(b)

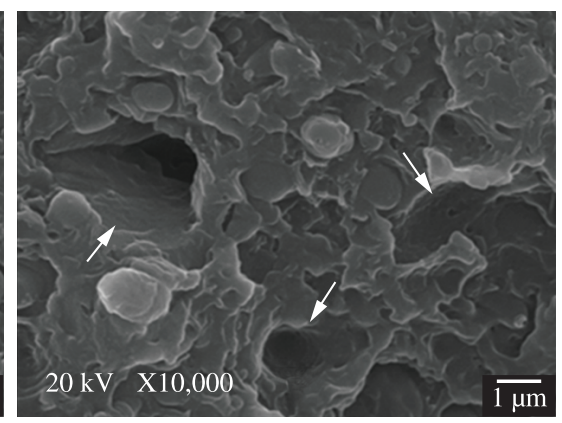

(c)

Figura 5. Micrografias obtidas por MEV das superficies fraturadas das composições com e sem copolímero tenacificador. a) HIPS reciclado sem adição de SBS; b) HIPS reciclado/SBS linear 85/15; and c) HIPS reciclado/SBS radial 85/15. As setas na Figura 5c exemplificam a formação de vazios por cavitação.

Tabela 2. Propriedades em tração e flexão, em função do teor e do tipo de SBS de blendas HIPS reciclado/SBS.

\begin{tabular}{ccccccc}
\hline Amostra & SBS & HIPS reciclado/SBS (\%massa) & RT (MPa) & $\varepsilon \mathbf{R}(\%)$ & RF (MPa) & Ef $(\mathbf{M P a})$ \\
\hline 0 & - & $100 / 0$ & $21,9 \pm 1,0$ & $4,5 \pm 2,4$ & $46,3 \pm 2,2$ & $2475 \pm 160$ \\
5L & Linear & $95 / 5$ & $21,8 \pm 0,9$ & $6,2 \pm 4,3$ & $42,1 \pm 1,0$ & $2169 \pm 44$ \\
10L & Linear & $90 / 10$ & $20,8 \pm 0,2$ & $20,5 \pm 3,7$ & $36,3 \pm 0,8$ & $1884 \pm 83$ \\
15L & Linear & $85 / 15$ & $18,4 \pm 0,3$ & $29,1 \pm 6,9$ & $31,1 \pm 1,4$ & $1483 \pm 162$ \\
5R & Radial & $95 / 5$ & $22,3 \pm 0,4$ & $16,7 \pm 4,0$ & $40,1 \pm 0,6$ & $2094 \pm 85$ \\
10R & Radial & $90 / 10$ & $22,2 \pm 0,5$ & $22,2 \pm 0,5$ & $32,5 \pm 0,3$ & $1483 \pm 25$ \\
15R & Radial & $85 / 15$ & $20,8 \pm 0,4$ & $28,7 \pm 4,3$ & $28,9 \pm 0,3$ & $1351 \pm 53$ \\
\hline
\end{tabular}

RT = Resistência à Tração; $\varepsilon R$ = Deformação na Ruptura; RF = Resistência à Flexão; Ef = Módulo de Elasticidade em Flexão. 

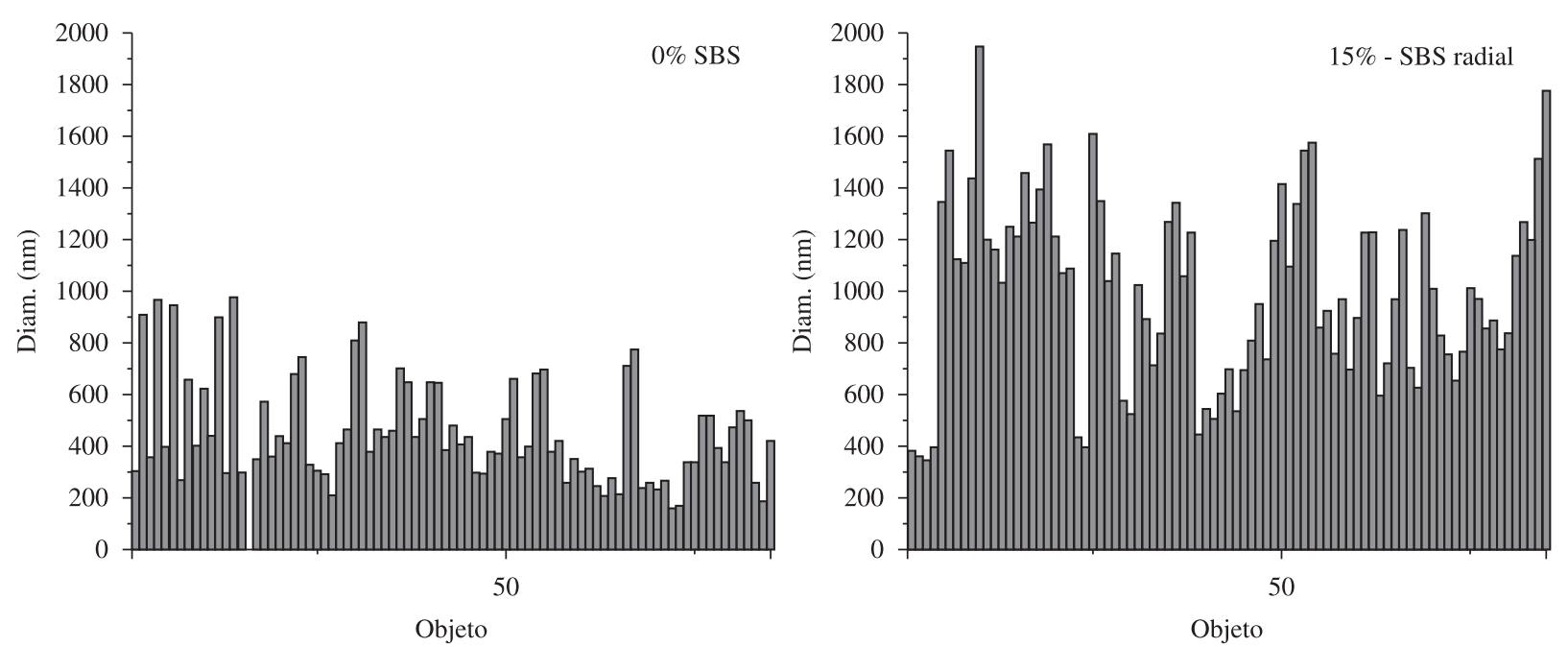

Figura 6. Distribuição de tamanho dos vazios das micrografias: 5 (a), HIPS reciclado $\bar{D}=420 \pm 190 \mathrm{~nm}$ e 5 (c) HIPS reciclado/ $15 \% \mathrm{SBS} \operatorname{radial} \bar{D}=1000 \pm 370 \mathrm{~nm}$.

Tabela 3. Resultados obtidos por termogravimetria para as blendas HIPS reciclado/SBS.

\begin{tabular}{cccccc}
\hline Amostra & SBS & $\begin{array}{c}\text { HIPS reciclado/ } \\
\text { SBS (\% massa) }\end{array}$ & $\begin{array}{c}\mathbf{T}_{\mathbf{0}} \\
\left({ }^{\circ} \mathbf{C}\right)\end{array}$ & $\begin{array}{c}\mathbf{T}_{\mathbf{0}, 5} \\
\left({ }^{\circ} \mathbf{C}\right)\end{array}$ & $\begin{array}{c}\text { Resíduo a } \\
\mathbf{6 0 0}\end{array}{ }^{\circ} \mathbf{C}(\boldsymbol{\%})$ \\
\hline 0 & - & $100 / 0$ & 401 & 462 & 6,1 \\
5L & Linear & $95 / 5$ & 403 & 459 & 5,0 \\
10L & Linear & $90 / 10$ & 402 & 459 & 4,1 \\
15L & Linear & $85 / 15$ & 411 & 469 & 4,5 \\
5R & Radial & $95 / 5$ & 415 & 466 & 6,2 \\
10R & Radial & $90 / 10$ & 411 & 470 & 4,6 \\
15R & Radial & $85 / 15$ & 417 & 473 & 4,5 \\
SBS Linear & Linear & $0 / 100$ & 439 & 475 & 3,3 \\
SBS Radial & Radial & $1 / 100$ & 417 & 487 & 1,5 \\
\hline
\end{tabular}

15\% de copolímero SBS sugerem que as partículas de copolímero são arrancadas da matriz de HIPS, por cavitação, formando vazios com rompimento na interface, indicando uma boa compatibilidade entre os componentes. O diâmetro médio dos vazios, evidentes nas micrografias de cada composição foi obtido pela medida de pelo menos 150 objetos independentes.

$\mathrm{O}$ aspecto da fratura do HIPS reciclado, sem a adição de copolímero, Figura 5a e Figura 6, mostra vazios de diâmetro médio $(\bar{D}=420 \pm 190 \mathrm{~nm})$, indicando uma provável degradação com redução do tamanho das partículas da fase butadieno, resultando na baixa resistência ao impacto do material. Segundo a literatura ${ }^{[2,15]}$, existe um tamanho de partícula de elastômero mais efetivo para a tenacificação do HIPS, sendo que partículas com diâmetro médio menor que $1 \mu \mathrm{m}$ são pouco eficientes na melhora da resistência ao impacto. Nas composições com SBS radial, Figura 5c e Figura 6, o tamanho médio dos vazios corresponde a um maior tamanho médio das partículas dispersas $(\bar{D}=1000 \pm 370 \mathrm{~nm})$, o que corrobora o resultado de maior resistência ao impacto obtida em todas as composições, quando comparada às composições com iguais teores de SBS linear, sugerindo que existe um tamanho médio de partículas mais efetivo para a tenacificação de poliestireno. A distribuição bimodal, com uma parte de partículas pequenas do elastômero e outra de partículas maiores dispersas na matriz é mais efetiva na resistência ao impacto do HIPS que a distribuição monomodal para um mesmo teor de borracha, pois as partículas pequenas iniciam as microfissuras, e as partículas maiores terminam e controlam a propagação das mesmas ${ }^{[2,15]}$.

\section{Conclusões}

A adição de copolímeros de SBS, de microestrutura radial ou linear, demonstrou ser uma técnica eficiente para a recuperação de propriedades mecânicas de HIPS reciclado.

Em todos os teores empregados $(5,10$ e $15 \% \mathrm{~m} / \mathrm{m})$, os valores de resistência ao impacto das misturas HIPS reciclado/SBS radial foram maiores que os encontrados para as misturas com SBS linear. A maior recuperação de propriedades de impacto foi verificada com a adição de $15 \%$ de SBS radial. Neste teor, verificou-se que a resistência ao impacto do HIPS reciclado aumento em três vezes, aproximadamente (de 24,6 J.m ${ }^{-1}$ para 74,7 J). Contudo, a adição de $15 \%$ de SBS linear também apresentou aumento versus o HIPS não modificado (de 24,6 J.m $\mathrm{m}^{-1}$ para 57,0 J).

Os ensaios de tração e flexão indicaram uma diminuição da rigidez de todas as composições com o aumento do teor de copolímero SBS observado pela diminuição do módulo elástico e aumento da deformação na ruptura. Esses resultados confirmam o que foi verificado nos resultados de resistência ao impacto, sendo que, dentre os copolímeros SBS empregados nesse estudo, o SBS radial foi mais efetivo na recuperação de propriedades do HIPS do que o SBS linear.

As micrografias das composições HIPS/SBS sugerem que as partículas de copolímero SBS foram arrancadas da matriz de HIPS reciclado, formando vazios com rompimento da interface. O tamanho maior dos vazios nas composições com SBS radial provavelmente está associado ao tamanho das partículas dispersas, efeitos de cavitação favorecidos pela estrutura tipo estrela e à maior massa molecular deste copolímero, o que, novamente, concorda com os valores de maior resistência ao impacto obtidos em todas as composições de SBS radial quando comparados aos das composições com iguais teores de SBS linear.

\section{Agradecimentos}

Os autores agradecem à Softer Brasil Compostos Termoplásticos Ltda e à Feevale pelo fornecimento de recursos e estrutura para o desenvolvimento deste trabalho.

\section{Referências Bibliográficas}

1. De Paoli, M. A. - "Degradação e Estabilização de Polímeros", Editora Artliber, São Paulo (2008). 
2. Grassi, V. G.; Camargo M. M. F. \& Dal Pizzol. M. F. - Polímeros,11, p.158 (2001). http://dx.doi.org/10.1590/S0104-14282001000300016

3. Rovere, J.; Correa, C. A.; Grassi, V. \& Dal Pizzol. M.F. - Polímeros,18, p.12 (2008). http://dx.doi.org/10.1590/S0104-14282008000100007

4. Yilmaz, T.; Sahin, T. \& Sinmazcelik, T. - J Mater Sci, 44, p. 4308 (2009). http://dx.doi.org/10.1007/s10853-009-3640-3

5. Associação Brasileira da Indústria do Plástico - ABIPLAST. - "O Perfil da Indústria Brasileira de Transformação de Material Plástico" (2009). Disponível em: <http://www.abiplast.org.br/upload/File/PERFIL2009/ PERFIL2009(1).pdf>. Acesso em: 27 nov. 2010.

6. Spinacé, M. A. S. \& De Paoli, M.A. - Química Nova, 28, p.65 (2005).

7. Zanin, M.; MANCINI, S. D. - "Resíduos Plásticos e Reciclagem: Aspectos Gerais e Tecnologia”, Editora da Universidade Federal de São Carlos, São Carlos (2004).

8. Compromisso Empresarial para Reciclagem - CEMPRE. Disponível em: <http://www.cempre.org.br/fichas_tecnicas.php?lnk=ft_plastico_ filme.php>. Acesso em: 27 nov. 2010.

9. Vilaplana, F.; Karlsson, S. \& Riber-Greus, A. - Eur. Polym. J., 43, p.4371 (2007). http://dx.doi.org/10.1016/j.eurpolymj.2007.07.017

10. Vilaplana, F.; Riber-Greus, A. \& Karlsson, S. - Macromol. Mater. Eng., 293, p. 274 (2008). http://dx.doi.org/10.1002/mame.200700393

11. Parres, F.; Balart, R.; López, J. \& García, D. - J. Mater Sci, 43, p.3203 (2008). http://dx.doi.org/10.1007/s10853-008-2555-8

12. Vilaplana, F. \& Karlsson, S. - Polym. Degrad. and Stability, 91, p.2163 (2006). http://dx.doi.org/10.1016/j.polymdegradstab.2006.01.007

13. Borrelly, D. F. - "Estudo Comparativo da Degradação de Poliestireno $e$ de Poliestireno de Alto Impacto por Envelhecimentos Natural e Artificial", Tese de Mestrado, Universidade de São Paulo, Brasil (2002).
14. Silva, P. A. - "Nanocompósitos de borracha termoplástica do tipo poli(estireno-b-butadieno-b-estireno)-SBS e poli(estireno-butadieno) $S B R$ ". Dissertação de Mestrado, Universidade Federal do Rio Grande do Sul, Brasil (2007).

15. Veronese, V. - "Desenvolvimento e Avaliação de Propriedades de Misturas de Poliestireno (PS) e Copolímeros em Bloco de EstirenoButadieno-Estireno (SBS)", Dissertação de Mestrado, Universidade Federal do Rio Grande do Sul, Brasil (2003)

16. Legge N. R. - Thermoplastic Elastomers, Hanser Publishers, New York (1996).

17. Rabelo, M. S. - “Aditivação de Polímeros”, Editora Artliber, São Paulo (2000).

18. Wunsch, J. R. - "Polystyrene: Synthesis, production and applications", Smithers, London (2000).

19. Canevarolo Junior, S. V. - "Técnicas de Caracterização de Polímeros", Artliber Editora Artlieber, São Paulo (2003).

20. Vilaplana, F.; Karlsson, S. \& Riber-Greus, A. - Polym Degrad. Stability, 95, p.172 (2010). http://dx.doi.org/10.1016/j. polymdegradstab.2009.11.033

21. Videolar. - "Poliestireno". Disponível em: <http://www.videolar. com.br/PDF/especificacoes $/ 4.5 .0001 \% 20-\% 20$ Ficha $\% 20$ T\%C3\%A9cnica\%20Poliestireno\%20HIPS\%20825-rev03.pdf>. Acesso em: 16 jun. 2011.

22. Petrobras. - "SR550 - Injeção Poliestireno Alto Impacto". Disponível em: <http://www.petrobras.com.ar/Innova/Internet_Innova/Portugues/ Productos_Servicos/Imagenes/SR550.pdf $>$. Acesso em: 16 jun. 2011.

Enviado: $28 / 11 / 10$

Reenviado: 05/07/11 Aceito: 07/08/11 